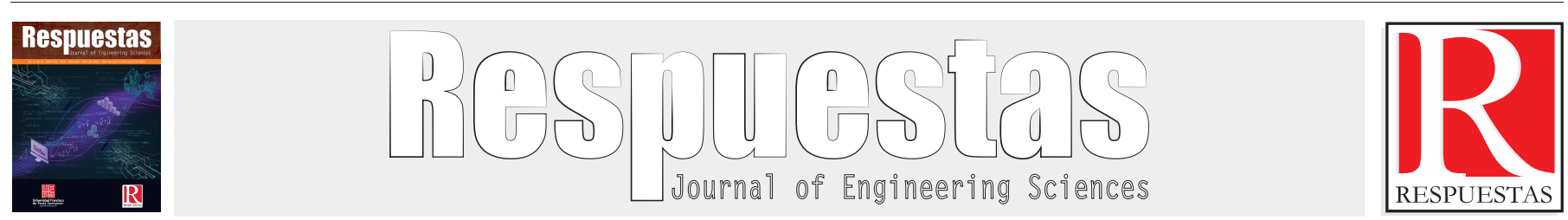

Artículo Original

https://doi.org/10.22463/0122820X.1498

\title{
Servicio web para la geolocalización de los vehículos de transporte público en la ciudad de Cúcuta
}

Web service for the geolocation of the public transport vehicles in the city of Cúcuta

Jhon Alejandro Castro-Correa, Sergio Basilio Sepúlveda-Mora, Byron Medina-Delgado, Dinael Guevara-Ibarra. ${ }^{\mathbf{d}}$

angeniero Electrónico, jhonalejandrocc@ufps.edu.co,orcid.org/0000-0002-2507-3535, Universidad Francisco de Paula Santander, Cúcuta, Colombia.

${ }^{b}$ Master of Science in Electrical and Computer Engineering, sergio.sepulveda@ufps.edu.co, orcid.org/0000-0002-1248-7616, Universidad Francisco de Paula Santander, Cúcuta, Colombia.

'Magister en Ingeniería Electrónica, byronmedina@ufps.edu.co, orcid.org/0000-0003-0754-8629, Universidad Francisco de Paula Santander, Cúcuta, Colombia.

'Doctor en Ingenieria, dinaelgi@ufps.edu.co, orcid.org/0000-0003-3007-8354, Universidad Francisco de Paula Santander, Cúcuta, Colombia.

Cómo citar: J.A. Castro-Correa, S.B. Sepúlveda-Mora, B. Medina-Delgado y D. Guevara-Ibarra, "Servicio web para la geolocalización de los vehículos de transporte público en la ciudad de Cúcuta", Respuestas, vol. 23, no. S1, pp. 29 - 37, 2018. https://doi.org/10.22463/0122820X.1498

Recibido: Enero 29, 2018; Aprobado: Junio 20, 2018.

\begin{tabular}{|c|c|}
\hline & RESUMEN \\
\hline $\begin{array}{l}\text { Palabras Clave: } \\
\text { Geolocalización } \\
\text { GPS } \\
\text { Servicio web } \\
\text { Servidor web } \\
\text { Transporte público }\end{array}$ & $\begin{array}{l}\text { En la ciudad de Cúcuta el medio de transporte que predomina para la movilización de personas entre un punto y otro es el } \\
\text { autobús tradicional, dicho servicio no ofrece a los usuarios la posibilidad de conocer de forma precisa el trayecto y el itinerario } \\
\text { de los autobuses que transitan por la ruta. Este trabajo está orientado a diseñar e implementar un servicio web que permita } \\
\text { visualizar en tiempo real las rutas y los vehículos de transporte público que circulan en la ciudad de Cúcuta. La geolocalización } \\
\text { de los autobuses se realizó mediante un GPS que proporciona el posicionamiento de los vehículos mediante el protocolo NMEA } \\
\text { 0183; esta información es almacenada en un servidor para luego ser enviada (usando PHP) y visualizada. La interfaz de usuario } \\
\text { se desarrolló a través de un sitio web funcional y de diseño adaptable para computadoras, tabletas y celulares utilizando HTML5, } \\
\text { CSS3 y JavaScript, donde dependiendo de la ruta escogida se realiza una petición al servidor para obtener los datos } \\
\text { correspondientes y mostrarlos en un mapa utilizando la API de Google Maps. Se implementó el servicio web permitiendo } \\
\text { visualizar las rutas y la posición del vehículo en tiempo real junto con distintas variables de interés como el estado de } \\
\text { movimiento o reposo, velocidad, rumbo y tiempo de llegada a cada una de las paradas preestablecidas en la ruta; asimismo } \\
\text { dentro del portal web se da la posibilidad de generar registros históricos concernientes a la ubicación y recorrido de cada autobús } \\
\text { que son visualizados mediante tablas y gráficas generadas por el usuario. }\end{array}$ \\
\hline
\end{tabular}

\section{ABSTRACT}

\section{Keywords:}

Geolocation

GPS

Web Service

Web server

Public transportation
In the city of Cucuta, the main way of transportation for the mobilization of people between two points is the traditional bus; currently, this service does not offer to users the possibility of knowing precisely the route and the itinerary of the buses that pass through it. This work is aimed to design and implement a web service that allows visualizing in real time the routes and public transport vehicles that circulate in the city of Cucuta. The geolocation of the buses was done through a GPS module that provides the positioning of the vehicles using the NMEA 0183 protocol; the information is stored on a server to be sent (using PHP) and viewed later. The user interface was developed as a functional website with adaptable design for computers, tablets, and cellphones using HTML5, CSS3, and JavaScript. Depending on the chosen route, a request is made to the server to obtain the corresponding data which is later displayed on a map using the Google Maps API. The web service allows to visualize the routes and the position of the vehicles in real time with several variables of interest such as the state of movement, speed, course, and time of arrival at each of the pre-established stops on the route; also, within the web portal is possible to generate historical records regarding the location and route of each bus, which are visualized through tables and graphs generated by the user.

\section{Introducción}

El transporte público se ha convertido en parte de la vida diaria, pues en la actualidad la mayoría de la población hace uso de este servicio para desplazarse de un lugar a otro, ya sea, dentro o fuera del casco urbano. En la ciudad de Cúcuta, los buses son distribuidos en diferentes rutas de transporte dentro del área metropolitana con el fin de cubrir la mayor cantidad de área. Cuando se hace uso del servicio es muy común que los usuarios deban emplear varios minutos de su tiempo esperando por su vehículo debido a problemas de tráfico [1], puesto que no tienen la posibilidad de visualizar ni estimar el tiempo que tarda el autobús en llegar a su estación o punto de embarque, esta problemática ha llevado a que se presenten numerosas propuestas en diferentes partes de mundo que sirven como alternativas de solución.

En [2], se propuso un sistema de rastreo basado en servicios de ubicación consciente y códigos QR, donde se utilizan herramientas como Google Transit aplicado a dispositivos móviles en Turquía.

Asimismo, en [3] se presentó un sistema de rastreo para buses basado en GPS utilizando una aplicación Android para visualizar la ruta junto a las posiciones del usuario y el bus, implementado en Patiala, India.

Por otra parte, en Sri Lanka, los autores en [4] desarrollaron un sistema en tiempo real para el rastreo de buses e itinerario utilizando sensores inalámbricos y tecnología móvil, obteniendo alta precisión de identificación a un bajo costo.

Este trabajo propone el desarrollo de un servicio web de diseño adaptable, sencillo y eficaz que permita mostrar en tiempo real la ubicación y ruta de los vehículos de transporte público en la

*Autor de correspondencia.

E-mail:dinaelgi@ufps.edu.co (Dinael Guevara Ibarra),

(c) (i) (3) $\odot$ Peer review is the responsibility of the Universidad Francisco de Paula Santander. (c) $(1)(-)$ This is an article under the license CC BY-NC-ND 
ciudad de Cúcuta; este dispone de una interfaz web, de la implementación y configuración de un servidor para recibir y enviar datos de coordenadas a una página web, así como el uso de bases de datos para almacenar registros históricos de la actividad de los vehículos para ser visualizados cuando se requiera. La elaboración del sitio se planeó teniendo en cuenta metodologías de diseño y usabilidad, además de tener en cuenta el uso software de código libre y lenguajes de programación con gran respaldo y aceptación por parte de la comunidad de desarrolladores.

El artículo se encuentra desarrollado como sigue: los materiales y métodos utilizados para el desarrollo del aplicativo web, desde la perspectiva de la interfaz del usuario hasta la configuración del servidor, los resultados obtenidos luego del desarrollo del software, y finalmente, las conclusiones y recomendaciones para futuras implementaciones de sistemas del mismo tipo.

\section{Materiales y métodos}

Un servicio web es un componente de software que implementa funciones concretas y que permite a las aplicaciones en desarrollo [5] y a las ya existentes exponer sus funcionalidades para que puedan ser utilizadas en todos los puntos donde se necesiten [6]. El aplicativo web presentado a continuación fue enfocado a la geolocalización de vehículos de transporte público, cuyo propósito es la ubicación de un objeto en un sistema de coordenadas determinado [7]; el software diseñado ha sido desarrollado para funcionar bajo el servidor HTTP Apache, que en la actualidad, es instalado y utilizado en gran parte de hostings alrededor del mundo, permitiendo una mayor aplicabilidad de la propuesta realizada. A continuación se describen los elementos utilizados para el diseño y desarrollo del servicio, así como la metodología utilizada para ello.

\section{Diseño del servicio}

Para el diseño y desarrollo se recurrió a la arquitectura de tres capas presentada en [8], la cual consta de la capas de presentación, proceso y de datos, tal y como se detalla en la Figura 1. En la capa de presentación (interfaz) se utilizaron los lenguajes HTML5 y CSS3 para el diseño de la interfaz de usuario, mientras que JavaScript y Jquery para la captura y validación de datos; en la misma capa (presentación), pero en el apartado de control, se implementó Ajax para el envío y recibimiento de peticiones asíncronas. A su vez, en la capa de negocios, se utilizó PHP para la consultas en el servidor e invocación de datos, todo esto, bajo el servidor Apache. Finalmente, en la capa de datos fue usado el gestor de bases de datos MySQL para el almacenamiento, recuperación y eliminación de información. Todo lo anterior teniendo en cuenta los estándares establecidos por el grupo W3 [9].

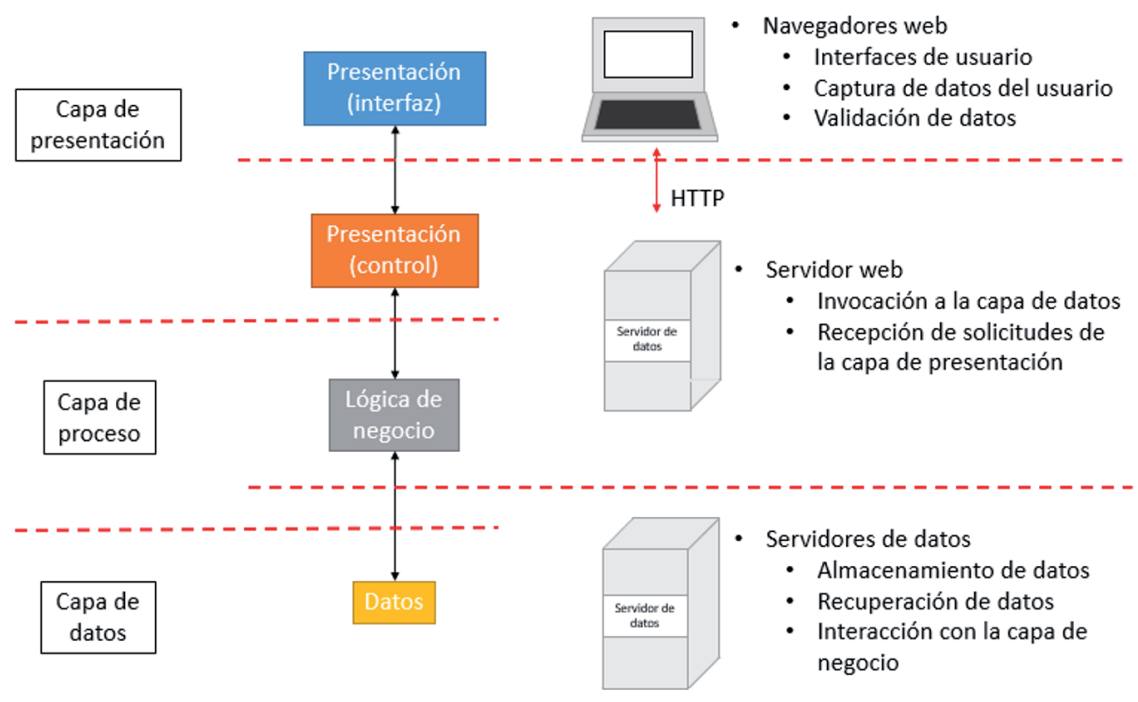

Figura 1. Arquitectura de 3 capas para el desarrollo de software

\section{Metodologia}

La metodología elegida para el desarrollo del servicio web fue el modelo en cascada, el cual se basa en actividades separadas y consecutivas que son definidas como: especificación (análisis y definición de requerimiento), implantación (diseño, codificación y validación) y mantenimiento [10]. Subsiguientemente, se describen las acciones realizadas dentro de cada uno de los aspectos de la metodología especificada anteriormente.

\section{Análisis y definición de requerimiento}

Identificación de problemas, oportunidades y objetivos: En secciones anteriores se ha detallado la problemática a solucionar, la cual tiene una profunda relación con el tráfico y el transporte público del área metropolitana de la ciudad de Cúcuta. El 
diseño, desarrollo e implementación de un sistema de geolocalización fue elegida como alternativa y solución a la problemática planteada, debido a su escalabilidad y usabilidad y utilidad. Se definió el objetivo de desarrollar un servicio web capaz generar registros y permitir la geolocalización de los vehículos de transporte público en la ciudad de Cúcuta para aportar al mejoramiento del tráfico y condiciones viales en el área metropolitana.

Determinación de los requerimientos de información: Para desarrollar el servicio web se definió la necesidad de obtener y operar datos en tiempo real de los vehículos a localizar geográficamente, contar un registro de sugerencias y contactos dentro del portal web y disponer de información para el manejo de cuentas usuario.

Con respecto a los datos de los vehículos, se tuvieron en cuenta las siguientes variables: un identificador para cada sentencia de datos, un identificador de vehículo, ruta a la cual pertenece, coordenadas espaciales (latitud y longitud), velocidad, fecha y hora actual, curso y orientación. Para el registro de sugerencias y contactos, se utilizaron datos para: la identificación de solicitud enviada, nombre, email, asunto y mensaje. Por último, para las cuentas de usuario se emplearon: el identificador, nombre y contraseña para el perfil. Todo esto puede verse reflejado en la Figura 2, en donde se muestran las tablas de datos que almacenaron la información relevante para el funcionamiento del sistema web (dentro de la base de datos, BD), cabe resaltar que en cada una de ellas el identificador (id), es utilizado como llave primaria.

\begin{tabular}{|c|c|c|}
\hline gpstracker & & \\
\hline id & & \\
\hline $\operatorname{tag}$ & & \\
\hline route & & \\
\hline lat & & \\
\hline Ing & gpscontact & \\
\hline speed & id & \\
\hline time & name & gpsuser \\
\hline date & email & id \\
\hline course & subject & user \\
\hline card & message & password \\
\hline
\end{tabular}

Figura 2. Requerimiento de información para el servicio web

Diagrama de casos de uso: Tras evaluar y analizar las necesidades del software se desarrolló el diagrama de casos de uso correspondiente. En la Figura 3 se observa una versión simplificada de éste, en el cual se muestran las funciones principales requeridas para el servicio web desarrollado.

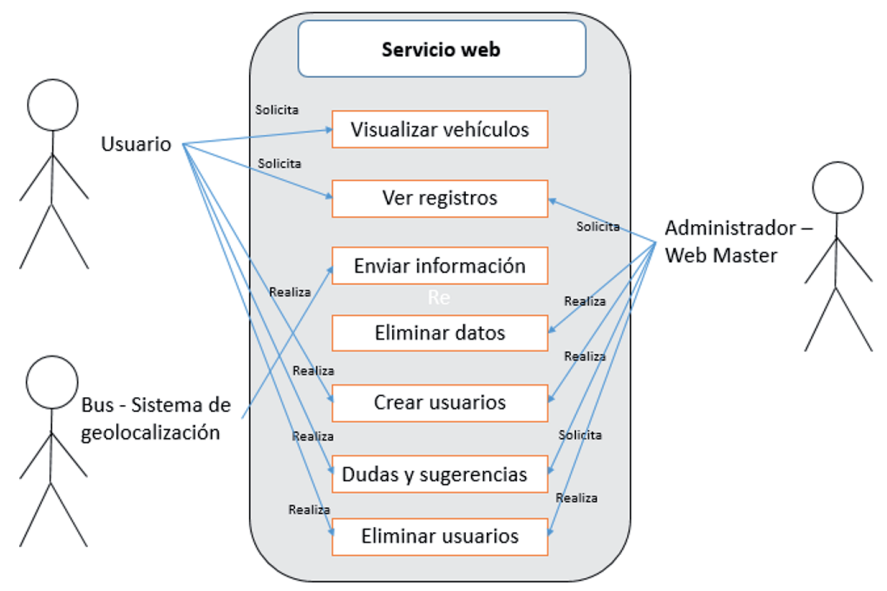

Figura 3. Diagrama de casos de uso simplificado usado para el servicio web

\section{Implantación}

Partiendo de la base de requerimientos y el propósito del servicio web, se dio paso a la etapa de diseño y desarrollo. En términos generales, la información referente a la localización de los buses en la ruta de transporte es enviada desde un GPS mediante peticiones del tipo GET (usando el estándar GPRS) para luego ser almacenada en una base de datos a través de funciones desarrolladas en PHP y ser mostrada en el navegador web. El esquema que muestra el funcionamiento general del sistema de monitorización se detalla en la Figura 4, donde se aprecia cada uno de los componentes que hacen parte de su funcionamiento.

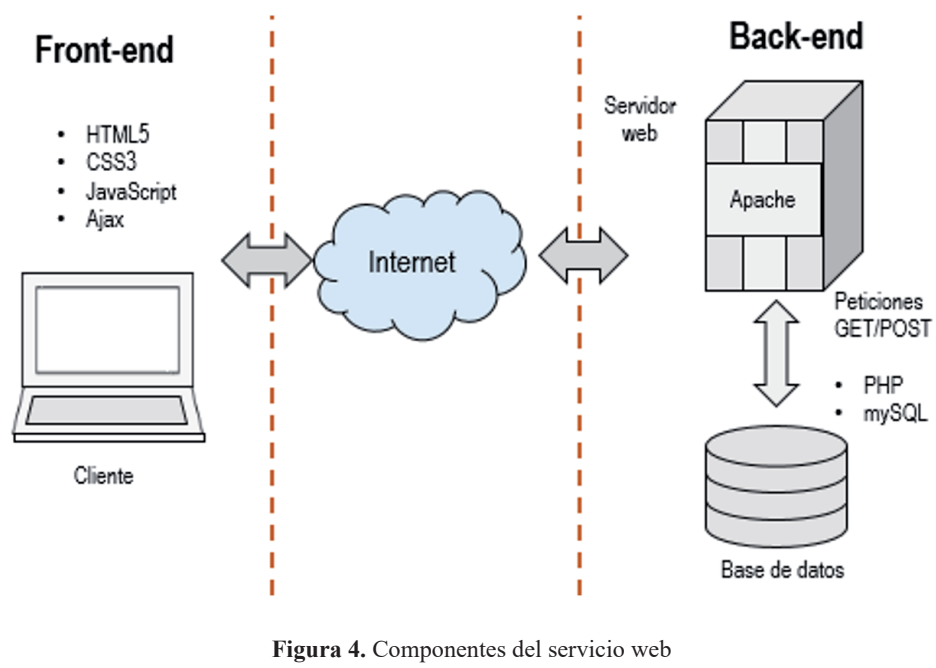

El diseño del portal web consta de dos partes fundamentales: el Back-end (algoritmos, funciones y programas ejecutados en el servidor web) y el Front-end (interfaz de usuario - códigos ejecutados y mostradas por el navegador). A continuación se detallan los componentes que hacen parte de la concepción y desarrollo del servicio, desde el punto de vista del Back-end y el Front-end. 
Back-end: Como se expuso al comienzo de la sección, el servidor web opera bajo Apache v2.4; dentro de este se utilizó mySQL como gestor de bases de datos. Mediante secuencias mySQL se crearon seis tablas independientes en la base de datos correspondientes a la información de los vehículos, datos de contacto, gráficas y cuentas de usuario, con el fin utilizar esta información para mejorar la experiencia de navegación por el sitio web.

Asi mismo, para realizar la comunicación entre el servidor y la página, se desarrollaron diferentes scripts para permitir la conexión, petición y exportación de registros desde la base de datos para mostrarlos mediante la herramienta correspondiente (mapas, gráficas o tablas). En la Figura 5 se observan las bases de datos elaboradas, mediante la herramienta phpMyAdmin.

\begin{tabular}{|c|c|c|c|c|c|c|c|c|c|c|c|c|}
\hline \multirow{4}{*}{$\begin{array}{c}\text { phpMyAdmII } \\
\text { Reciente Favoritas }\end{array}$} & \multicolumn{12}{|c|}{ 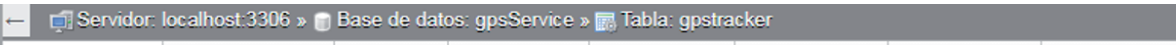 } \\
\hline & \multirow{2}{*}{$\begin{array}{l}\text { 曾 Examinar } \\
\leftarrow \text { ' } \rightarrow\end{array}$} & Estructura & $\square$ & & Q & Buscar & 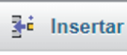 & \multicolumn{2}{|c|}{ Exportar } & Importar & \multicolumn{2}{|c|}{ a: Privileg } \\
\hline & & $\nabla$ & id & $\operatorname{tag}$ & route & lat & Ing & speed & time & date & course & card \\
\hline & $\square$ Editar & 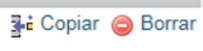 & 1 & 3 & 2 & 7.5243 & -72.5443 & 36.4 & 05:08:00 & $2017-08-22$ & 35.2 & ENE \\
\hline $\begin{array}{l}\text { - Nueva } \\
\text { gpsService }\end{array}$ & $\square$ Editar & z̧ Copiar $\odot$ Borrar & 2 & 3 & 2 & 7.5243 & -72.7443 & 36.4 & 05:08:00 & $2017-08-22$ & 35.2 & ENE \\
\hline L圆 Nueva & $\square$ Editar & 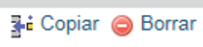 & 3 & 3 & 1 & 7.5243 & -72.9443 & 36.4 & 05:08:00 & $2017-08-22$ & 35.2 & ENE \\
\hline $\begin{array}{l}+12 \text { bus } \\
+-2 \text { buslist }\end{array}$ & $\square$ Editar & zjic Copiar $\odot$ Borrar & 4 & 3 & 1 & 7.5243 & -72.2443 & 36.4 & 05:08:00 & $2017-08-22$ & 35.2 & ENE \\
\hline$+-1-$ gpscontact & $\square$ Editar & żz: Copiar $\odot$ Borrar & 5 & 3 & 1 & 7.5243 & -72.9443 & 36.4 & 05:08:00 & 2017-08-22 & 35.2 & ENE \\
\hline +- gpstracker & $\square$ Editar & jat Copiar $\odot$ Borrar & 6 & 3 & 2 & 7.7243 & -72.5443 & 36.4 & 05:08:00 & $2017-08-22$ & 35.2 & ENE \\
\hline 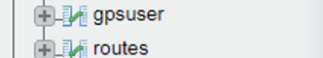 & $\square$ Editar & zj: Copiar $\odot$ Borrar & 7 & 3 & 1 & 7.9243 & -72.5443 & 36.4 & 05:08:00 & $2017-08-22$ & 35.2 & ENE \\
\hline +_[information_schema & $\square$ Editar & zit: Copiar @ Borrar & 8 & 3 & 1 & 7.9243 & -72.5443 & 36.4 & 05:08:00 & $2017-08-22$ & 35.2 & ENE \\
\hline T-10 mysql & $\square$ Editar & z̧子 Copiar $\odot$ Borrar & 9 & 3 & 1 & 7.7243 & -72.5443 & 36.4 & 05:08:00 & 2017-08-22 & 35.2 & ENE \\
\hline +10 performance_schema & $\square$ Editar & jat Copiar $\odot$ Borrar & 10 & 3 & 1 & 7.5243 & -72.5443 & 36.4 & 05:08:00 & $2017-08-22$ & 35.2 & ENE \\
\hline †-10 phpmyadmin & $\square$ Editar & zat Copiar $\odot$ Borrar & 11 & 1 & 2 & 7.6243 & -72.5443 & 36.4 & 05:08:00 & $2017-08-22$ & 35.2 & ENE \\
\hline & $\square$ Editar & zit: Copiar @ Borrar & 12 & 1 & 2 & 8.6243 & -82.5443 & 36.4 & 05:08:00 & $2017-08-22$ & 35.2 & ENE \\
\hline & $\square$ Editar & 굴: Copiar $\odot$ Borrar & 13 & 1 & 2 & 8.6243 & -82.5443 & 36.4 & 05:08:00 & $2017-08-22$ & 35.2 & ENE \\
\hline & $\square$ Editar & z̧jt Copiar $\odot$ Borrar & 14 & 1 & 2 & 8.6243 & -82.5443 & 36.4 & 05:08:00 & $2017-08-22$ & 35.2 & ENE \\
\hline
\end{tabular}

Figura 5. Bases de datos para la gestión del servicio web

Front-end: Para la interfaz de usuario se desarrollaron diferentes secciones, claramente establecidas y diferenciadas teniendo en cuenta un diseño adaptativo capaz de funcionar eficientemente en cualquier dispositivo [11].

En cuanto a la aplicación del cliente, se utilizó la API JavaScript de Google Maps con el fin de mostrar la ubicación de los vehículos en tiempo real, así como las rutas de transporte a las cuales pertenecen. La implementación de esta característica se realizó mediante JavaScript y Jquery. Del mismo modo, se implementaron peticiones asíncronas por medio de Ajax para obtener la información en tiempo real concerniente a la posición de los vehículos y rutas monitorizadas dentro del sitio web.

En la Figura 6 se muestra el diagrama de flujo que detalla el proceso de muestra de coordenadas geográficas en el mapa, en este se observa que solo se realizan las peticiones al servidor mientras alguna de las rutas de transporte es seleccionada dentro del menú del mapa; en caso que una ruta sea elegida se realizan peticiones en forma continua al servidor para obtener el último registro de la base de datos, correspondiente a los vehículos de la ruta escogida, para eventualmente mostrarlos en el mapa mediante marcadores y la API de Google Maps.

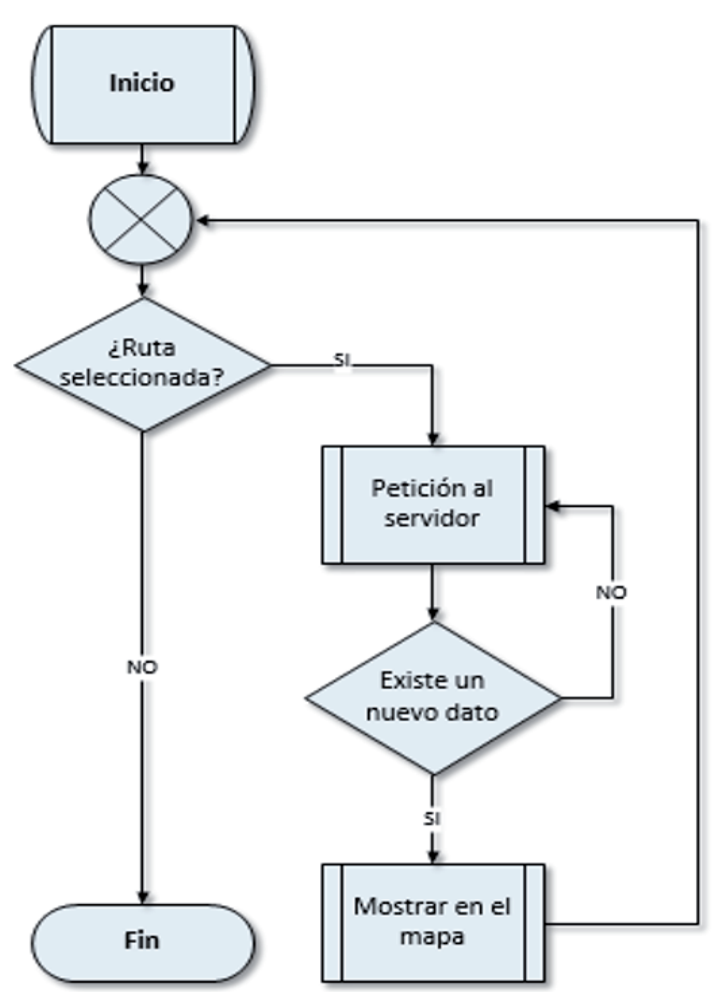

Figura 6. Diagrama de flujo para la muestra de datos en el mapa 
Para visualizar los resultados de consultas y tablas generadas desde la base de datos se hizo uso de la herramienta de Google Charts, la cual permite mostrar información proveniente del servidor en formato JSON en diferentes tipos de gráficas; para su integración se requirió del uso de JavaScript de lado del cliente y PHP para el servidor. De manera similar, se implementaron gráficas en tiempo real para mostrar la cantidad total de datos provistos por ruta y autobús, así como la velocidad actual de cada uno de los vehículos; para las gráficas en tiempo real se realizaron peticiones continuas al servidor, tratadas mediante scripts en PHP, cuyo propósito era llevar a cabo la consulta a la base de datos, obtener el último dato guardado en esta y mostrarlo en la gráfica en intervalos de tiempo periódicos (cada 20 segundos).

Aspectos de diseño: El desarrollo del portal web se definió teniendo en cuenta los siguientes conceptos: usabilidad, accesibilidad, aspectos técnicos y estéticos, eficiencia y seguridad [12].

Según Jakob Nielsen [13], la usabilidad es definida como un atributo de calidad de un sitio web, que determina la factibilidad de la interfaz para ser utilizada, también se refiere a los métodos para mejorar la facilidad de uso [14]. En este punto se desarrolló una interfaz sencilla y de fácil uso, donde el usuario tiene la posibilidad de navegar por secciones claramente delimitadas y de fácil comprensión.

Por otra parte, la accesibilidad es definida como la facilidad de un sitio web para ser accedido y usado por la mayor cantidad de personas, indiferentemente de las limitaciones del usuario [15]. Teniendo en cuenta lo anterior, la página web fue diseñada bajo la técnica de diseño responsive design, esto es, permite la adaptación y presentación del contenido en diferentes resoluciones de pantalla, lo que hace posible el acceso a la información desde cualquier dispositivo: computador de escritorio, laptops, tabletas y celulares.

Respecto al aspecto técnico y estético se tuvieron en cuenta diferentes características como títulos, enlaces, íconos, tipogra- fía, botones, estilo y lenguaje para obtener un diseño claro, sencillo y atractivo para la vista del usuario. Además, se consideró la psicología de colores para el diseño del sitio web, se utilizaron colores suaves y monocromáticos que reflejaran un ambiente relajante y refrescante para los usuarios hagan uso del portal.

Por último, en términos de seguridad se obtiene que el sitio ofrece al usuario una navegación mediante HTTPS, esto significa que el servidor cuenta con un certificado SSL que permite la navegación encriptada a través de la página, salvaguardando así la seguridad y los datos de los visitantes del sitio.

\section{Mantenimiento}

El servicio web fue puesto en marcha en un servidor físico dedicado que consta de un sistema embebido Raspberry Pi 3, bajo el sistema operativo Debian Stretch lite y Apache 9 como servidor web instalado. A partir de las pruebas y funcionamiento del servicio, se corrigieron errores y mejoraron los algoritmos desarrollados dentro de la etapa de negocios, todo con el fin de dar una mayor robustez a los componentes de software desarrollados.

\section{Resultados y análisis}

Luego de las fases de diseño y desarrollo se obtuvo una página web funcional, donde se permite al usuario navegar por medio de las diferentes secciones: contacto, preguntas frecuentes, ingreso/registro de usuario y el mapa interactivo; este última es la parte fundamental del servicio y permite la visualización de los vehículos en tiempo real, así como las rutas de transporte correspondientes. La interfaz desarrollada es clara, sencilla e intuitiva, dando al visitante datos y estadísticas sobre el servicio que se presta, así como instrucciones y accesos para ingresar a la aplicación de mapa interactivo.

En la Figura 7 se observa la página de inicio, la cual ofrece al usuario las pautas para hacer uso del servicio.

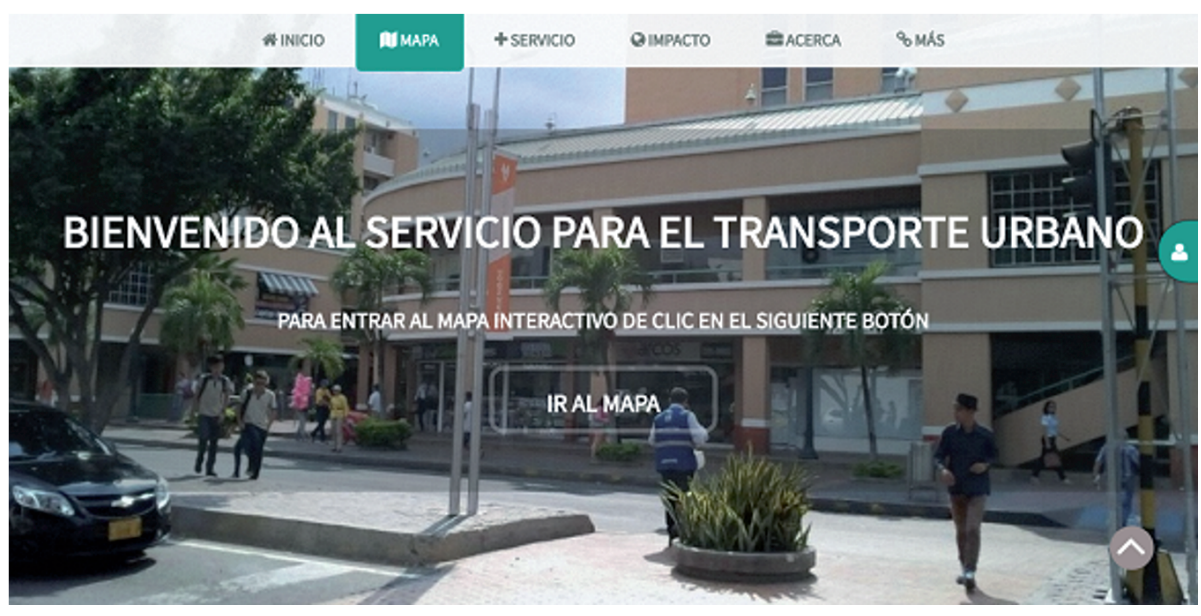

Figura 7. Página de inicio desarrollada 
Por su parte la interfaz del mapa permite al usuario seleccionar una o varias rutas de interés, mostrando en tiempo real la ubicación de los vehículos que circulan por dicho trayecto; además, provee datos de interés como la velocidad del bus y la posición del vehículo y tiempo de llegada a la parada más cercana por medio de un infowindow dentro del mapa. En la Figura 8 se muestra la sección de mapas dentro del sitio web.

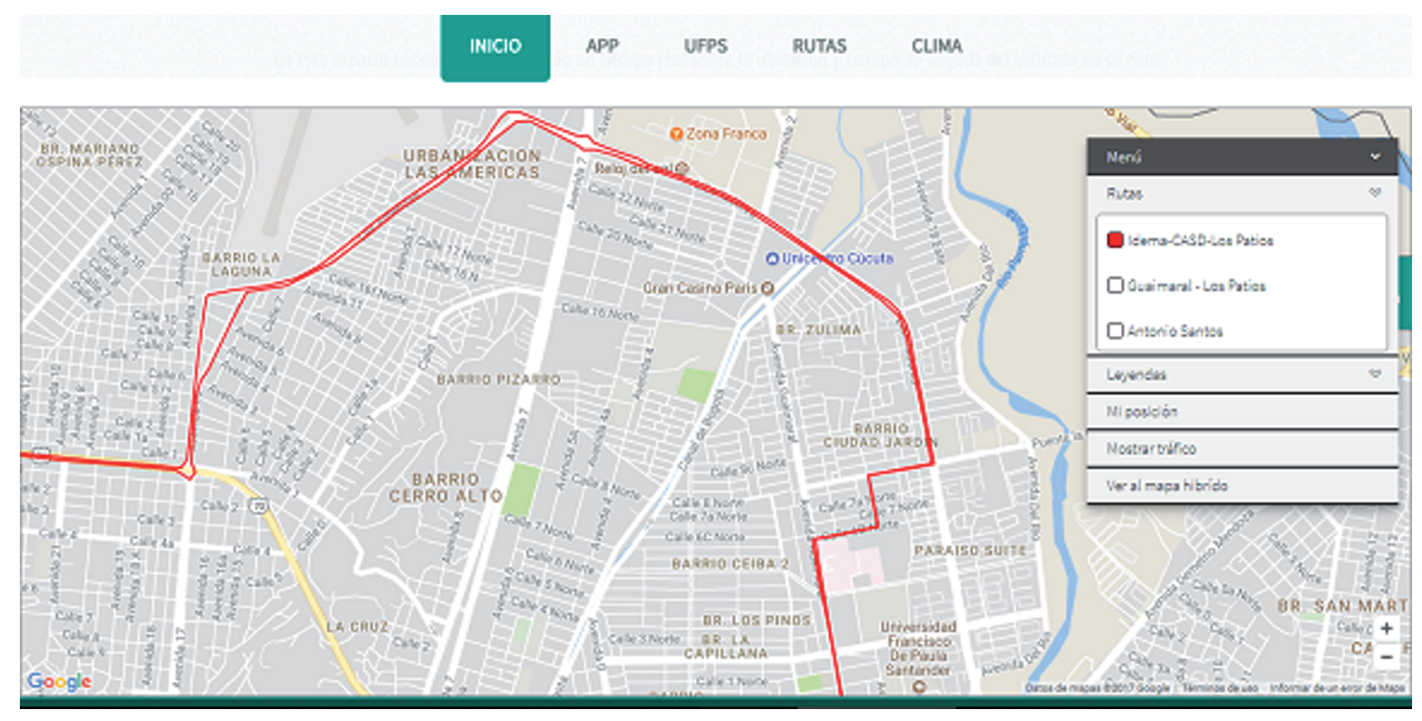

Figura 8. Interfaz de mapa en tiempo real

El menú lateral posee varias opciones entre las que se encuentran la selección de rutas, listado de leyendas que permiten la identificación de diferentes símbolos que aparecen en el mapa, la georreferenciación del usuario; es decir, la obtención las coordenadas y la posición actual del usuario, la opción de tráfico en vivo y el cambio de tipo de mapa.

Adicionalmente, en el sitio web se implementó la opción de crear cuentas y registros de usuarios, esta funcionalidad permite a los visitantes registrados obtener registros históricos de los parámetros de cada autobús (velocidad, rumbo, posición, tiempo y hora del registro), además permite la visualización de gráficas de velocidad en tiempo real de los vehículos pertenecientes a las rutas de transporte. En la Figura 9 se muestra la interfaz de ingreso de usuario.

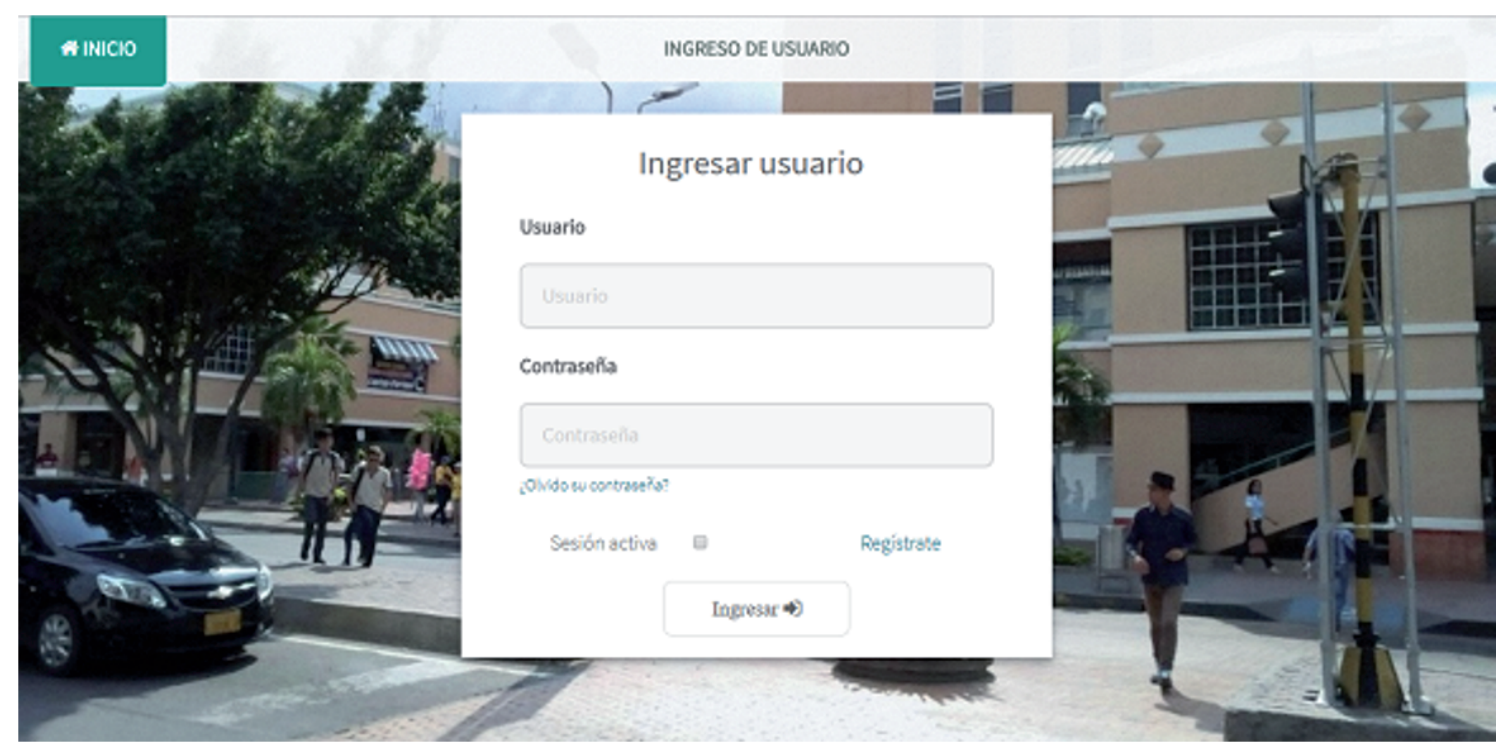

Figura 9. Interfaz de registro de usuario 
El registro de usuarios se realizó mediante PHP; utilizando cookies y variables de sesión se permite gestionar una sesión activa incluso después de cerrar la pestaña correspondiente. En términos de seguridad, la contraseña de cada usuario fue encriptada mediante la técnica de hash con el fin de evitar el robo de cuentas registradas en la plataforma; el método utilizado implica la encriptación de la contraseña junto con el identificador correspondiente a cada cuenta, el cual es la llave primaria de la base de datos realizada, de este modo la contraseña es inaccesible incluso para algún intruso dentro de la base de datos haciendo casi imposible la obtención de esta. En la Figura 10 se observa la gráfica en tiempo real sobre la velocidad de los vehículos, está es accesible por medio de cuenta de usuario.

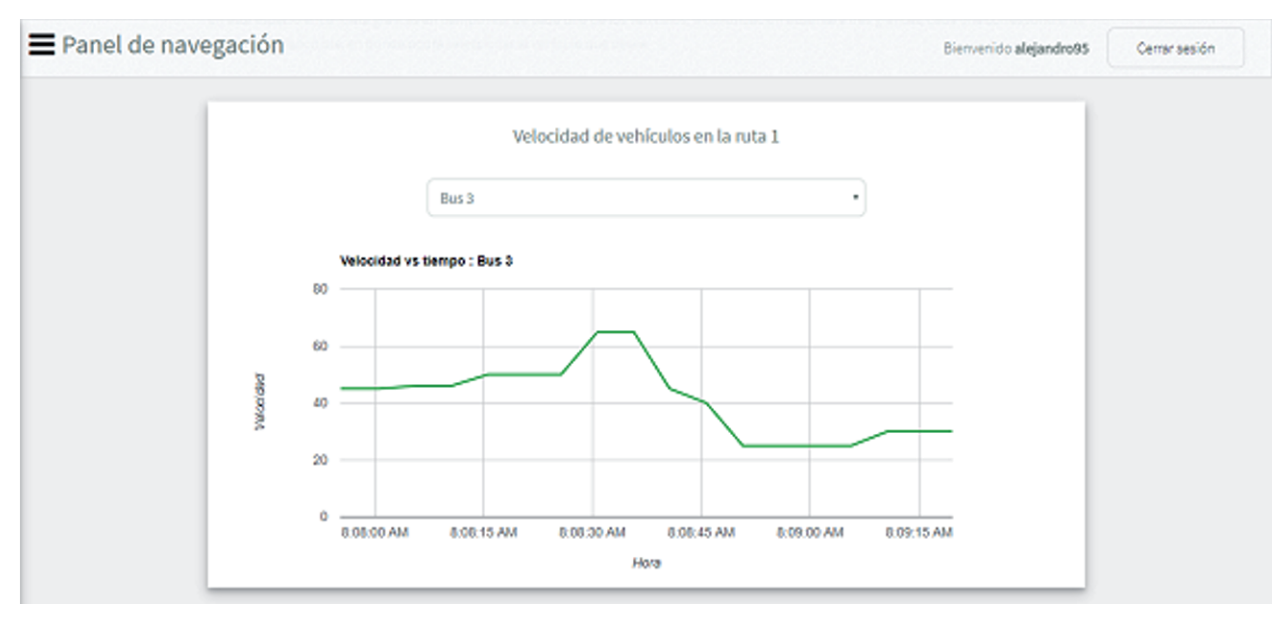

Figura 10. Gráfica en tiempo real de la velocidad del vehículo

Dentro del panel de navegación existen tantas gráficas como rutas disponibles esté soportando el servicio en ese momento, en cada gráfica se puede filtrar la búsqueda dependiendo de los buses disponibles, para luego mostrar los resultados; para el caso de la Figura 10 se seleccionó el tercer bus correspondiente a la primera ruta de transporte. Otra funcionalidad que soporta el panel es visualizar, mediante tablas, registros históricos de cada uno de los buses y/o rutas de transporte. En la Figura 11 se muestra la interfaz realizada para filtrar los resultados.

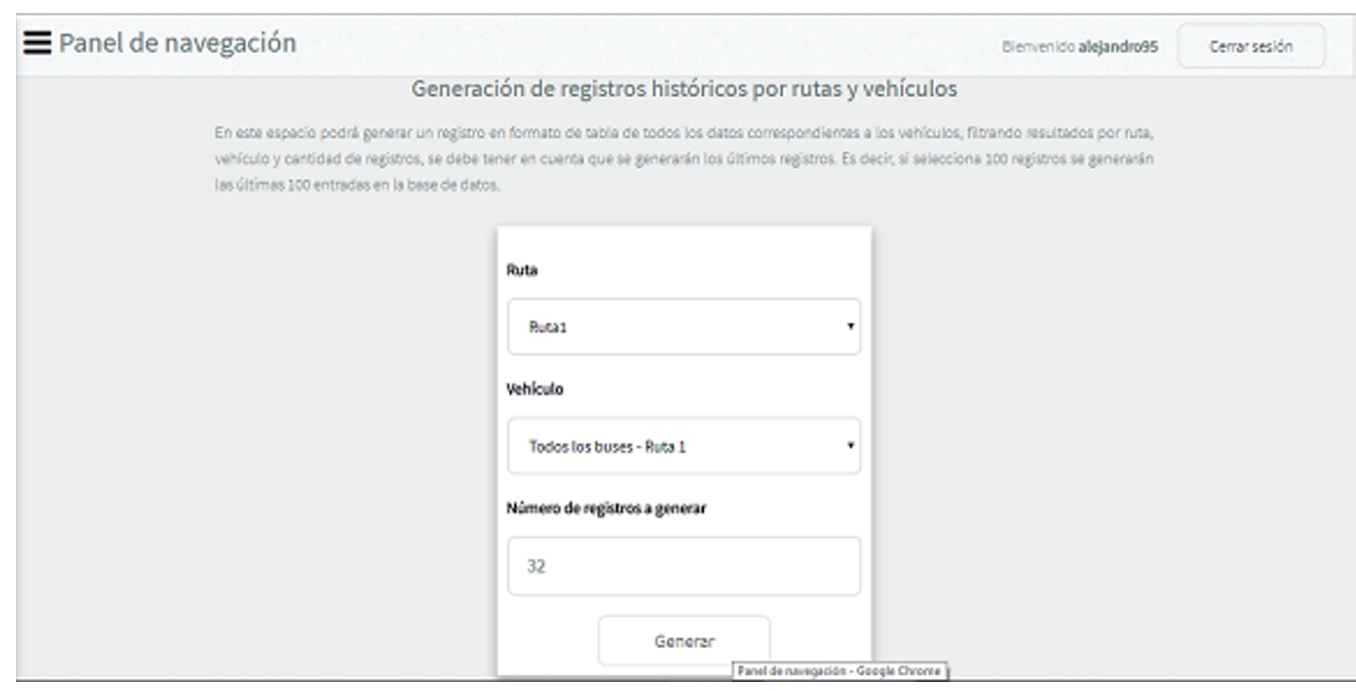

Figura 11. Interfaz para la generación de tablas dinámicas

Los registros pueden ser generados por rutas y vehículos, donde se puede discriminar si se generan los registros de una única ruta o si son todos los vehículos los que se deban mostrar en la tabla; adicionalmente se puede controlar la cantidad de registros a generar mediante un campo de tipo numérico. El tipo de generación es de orden descendente, desde el último dato almacenado en la base de datos, esto significa que si se realiza una consulta referente a 100 registros se mostrarán los últimos registros correspondientes a esta cantidad. La tabla generada muestra los valores correspondientes al número del bus (en la ruta), la ruta, longitud, latitud, velocidad, hora, fecha y rumbo del vehículo en ese instante. Lo anterior puede verse reflejado en la Figura 12, en donde se realizó la consulta con los datos mostrados en la Figura 11. 


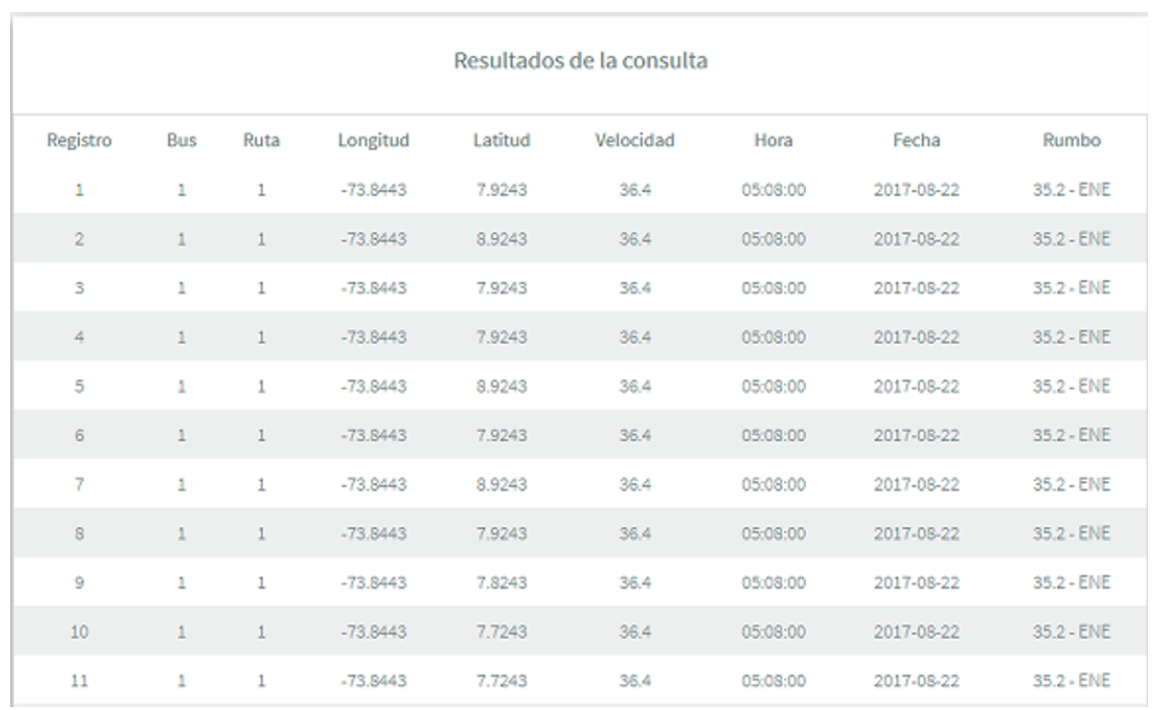

Figura 12. Tabla generada dentro del panel de navegación

\section{Conclusiones}

Se obtuvieron resultados en tiempo real sobre la localización de vehículos de transporte público en la ciudad de Cúcuta, mediante un servicio web sencillo y fácil de usar, el cual da acceso a información referente a los buses como posición, velocidad, rumbo y hora.

El servicio web es implementado bajo estándares de código abierto; todos los recursos utilizados como compiladores, APIs, software son de libre acceso, esto quiere decir que no se requieren licencias para el desarrollo del aplicativo, permitiendo que sea un servicio de muy bajo costo para su implementación.

Para la realización del servicio web fue necesario considerar las limitaciones en la cuota de manejo de la API de Google Maps, pues esta permite 25.000 cargas en el mapa gratuitas por día. Se tuvo en cuenta el tiempo de navegación por usuario además del tiempo en el cual muestra la información de cada vehículo en el mapa con el fin de hacer la web lo más eficiente posible.

Para el funcionamiento del servicio web es necesario tener un sistema de rastreo satelital, con la capacidad de tomar los datos de posición, velocidad, fecha, hora y rumbo y enviarlos mediante HTTP/HTTPS al servidor mediante peticiones del tipo GET o POST para ser tratados y mostrados en la página web.

\section{Referencias}

[1] P. Dhruv, S. Rahul y M. Vikas, «Real-Time Bus Tracking System,» International Research Journal of Engineering and Technology (IRJET), vol. 4, pp. 743-746, Marzo 2017.
[2] S. Eken y A. Sayar, «A smart bus tracking system based on location-aware services and QR codes,» 2014 IEEE International Symposium on Innovations in Intelligent Systems and Applications (INISTA) Proceedings, pp. 299-303, Junio 2014.

[3] L. Singla y P. Bhatia, «GPS based bus tracking system,» 2015 International Conference on Computer, Communication, and Control (IC4), pp. 1-6, Septiembre 2015.

[4] A. H. SL y S. N. Samsudeen, «Real Time Bus Tracking and Scheduling System Using Wireless Sensor and Mobile Technology,» Journal of Information Systems and Information Technology, vol. 1, pp. 18-23, 2016.

[5] J. Quiñónez, V. Garzón, F. Martínez y J. C. Corrales, «Selección heurística de mecanismos de compresión aplicados a mensajes Soap para mejorar el acceso a servicios Web desde redes de baja capacidad,» El Hombre y la Máquina [en linea] 2012, pp. 65-78, 2012.

[6] L. M. Santos, D. W. Rico y A. A. Rincón, «Servicios web en telefonía celular,» Scientia Et Technica, vol. 15, pp. 363-368, 2009.

[7] S.Y. Velazco-Flórez y L. Joyanes-Aguilar, "Herramienta GIS y servicios web en la geolocalización como instrumento en la adecuada gestión del territorio: Geoportal IDE Chinácota", Respuestas, vol. 18, no. 1, pp. 50-67, 2013.

[8] H. D. Molina, E. Wainerman, L. A. Olsina Santos, T. Ballari y E. Echeverría, «Arquitectura Centrada en la Web para el control y Monitoreo de Funcionalidad Domótica,» de VI Congreso Argentino de Ciencias de la Computación, Comodoro Rivadavia, 2001. 
[9] R. Hernández Claro y D. Greguas Navarro, «Estándares de Diseño Web,» Ciencias de la Información, vol. 41, pp. 69-71, 2010.

[10] J. Cervantes Ojeda y M. Gómez Fuentes, «Taxonomía de los modelos y metodologías de desarrollo de software más utilizados,» Universidades, nº 52, pp. 37-47, 2012.

[11] Y. Manso Guerra, R. Cañizares González y J. P. Febles, «Diseño web adaptativo para la plataforma educativa ZERA,» Revista Cubana de Ciencias Informáticas, pp. 100-115, 2016.

[12] G. R. Solarte Martínez y C. A. Ocampo Sepulveda, «Usabilidad en espacio web,» Scientia Et Technica, vol. 16, pp. 251-256, 2010.

[13] C. Gutiérrez, «Diseño web y arquitectura de información para sitios 2.0,» Cuadernos de Información, pp. 58-65, 2008.

[14] G. Benigni, J. A. Ordaz, O. Gervasi y S. Pallottelli, «Usabilidad ágil y reingeniería de sitios web: Usabagle web,» SABER. Revista Multidisciplinaria del Consejo de Investigación de la Universidad de Oriente, vol. 23, pp. 51-61, 2011.

[15] L. Perurena Cancio y M. Moráguez Bergues, «Usabilidad de los sitios Web, los métodos y las técnicas para la evaluación,» Revista Cubana de Información en Ciencias de la Salud, vol. 24, pp. 176-194, 2013. 